\title{
Análisis de la dieta de Diplomystes (Siluriformes: Diplomystidae) de Chile
}

\section{Dietary analysis of Diplomystes (Siluriformes: Diplomystidae) from Chile}

\author{
Beltrán-Concha M..$^{1 *}$, Muñoz-Ramirez C. ${ }^{2}$, Ibarra J. ${ }^{1} \&$ Habit E. ${ }^{1,3}$ \\ ${ }^{1}$ Centro de Ciencias Ambientales EULA-Chile, Unidad de Sistemas Acuáticos, Universidad de Concepción, Concepción, \\ Chile. \\ ${ }^{2}$ Department of Ecology and Evolutionary Biology, Museum of Zoology, University of Michigan, Ann Arbor, MI 48109-1079, \\ USA. \\ ${ }^{3}$ Centro de Investigaciones en Ecosistemas Patagónicos, CIEP. Coyhaique, Chile. \\ *E-mail: mbeltrancon@gmail.com
}

\begin{abstract}
RESUMEN
Diplomystidae es reconocida como la familia más primitiva en el orden Siluriformes. En Chile se encuentran tres de las seis especies descritas (género Dyplomystes), las que se distribuyen alopátricamente desde la cuenca del río Rapel hasta la cuenca del río Valdivia $\left(34^{\circ} \mathrm{S}-40^{\circ} \mathrm{S}\right)$, más una población sin identificar a nivel específico en la cuenca del río Baker ( $\left.47^{\circ} \mathrm{S}\right)$. En este trabajo se estudia el contenido estomacal de poblaciones colectadas en todas las cuencas hidrográficas donde se encuentra actualmente la familia en Chile, incluyendo las poblaciones del río Baker. Se analizó un total de 175 individuos de los cuáles el 90,3\% fueron juveniles. Los resultados muestran que, independiente de la procedencia, el principal ítem en la dieta lo constituyen las larvas de dípteros de la Familia Chironomidae. La excepción fueron individuos del río Toltén que se caracterizaron por consumir mayoritariamente Simulidae en lugar de Chironomidae. Tampoco se encontraron marcadas diferencias entre la dieta de juveniles y adultos, a excepción de la presencia exclusiva de decápodos del género Aegla en estos últimos. Estos resultados corroboran una dieta carnívoro-generalista, sin modificaciones a lo largo del gradiente latitudinal. Concluimos que los peces del género Diplomystes consumen los mismos grupos funcionales de invertebrados acuáticos a lo largo de su distribución latitudinal en Chile.
\end{abstract}

Palabras claves: Contenido estomacal. Diplomystes. Centro y Centro Sur de Chile. Río Baker.

\begin{abstract}
Diplomystidae is well known as the oldest catfish family in the order Siluriformes. In Chile three of the six described species are present (genus Dyplomystes), distributed allopatrically in rivers from the Rapel Basin to the Valdivia Basin $\left(34^{\circ} \mathrm{S}-\right.$ $\left.40^{\circ} \mathrm{S}\right)$, and some isolated populations of unidentified diplomystids in the Baker Basin $\left(47^{\circ} \mathrm{S}\right)$. In this study we analyze the stomach content of individuals of Diplomystes from all hydrological drainages in which populations are currently present in Chile, including the Baker River. A total of 175 individuals were analyzed from which $90.3 \%$ were juveniles. Results show that, regardless of the provenance of individuals analyzed, larvae of the insect family Chironomidae were the most consumed item. The only exception occurred in the Toltén Basin, whose population consumed mostly larvae of the insect family Simulidae. No marked differences were found between the diet of juveniles and adults, except for the presence of crustaceans of the genus Aegla in adults. These results confirm a carnivore and generalist diet for the genus Diplomystes, with no changes along the latitudinal gradient in Chile.
\end{abstract}

KeYwords: Stomach content. Diplomystes. South and South-Central Chile. Baker River.

\section{INTRODUCCIÓN}

La familia Diplomystidae es la familia de peces más primitiva del orden Siluriformes (Arratia 1983, 1987, 1992; Grande 1987; de Pinna 1998; Sullivan et al. 2006) y endémica de la subregión Austral de América del Sur
(Arratia 1987). En Chile se encuentran 3 de sus 6 especies, las que se distribuyen alopátricamente en sentido latitudinal, en las hoyas hidrográficas de Chile central y sur, desde la cuenca del río Aconcagua hasta la cuenca del río Valdivia $\left(34^{\circ} \mathrm{S}-40^{\circ} \mathrm{S}\right)$ (Arratia 1983, 1987; Campos 1985; Habit et al. 2009). Sin embargo, la falta de registros en las cuencas 
del Aconcagua y Maipo durante los últimos 50 años sugiere su extinción local en ellas (Arratia 1987; Muñoz-Ramírez et al. 2010). De acuerdo a la literatura más reciente, Diplomystes chilensis (Molina 1782) se encontraría actualmente sólo en la cuenca del río Rapel (Campos et al. 1998; Dyer et al. 2000); la especie D. nahuelbutaensis Arratia 1987 se distribuiría desde la cuenca del Maule a la del Imperial (Arratia 1987; Habit 1994; Vila et al. 1996) y la especie D. camposensis Arratia 1987 se encontraría sólo en la Cuenca del río Valdivia (Arratia 1987; Campos et al. 1998; Habit et al. 2009). Además, se han registrado recientemente poblaciones de Diplomystes sin identificar a nivel específico en las cuencas de los ríos Mataquito, Toltén y Baker (Muñoz-Ramírez et al. 2010) (Fig. 1).

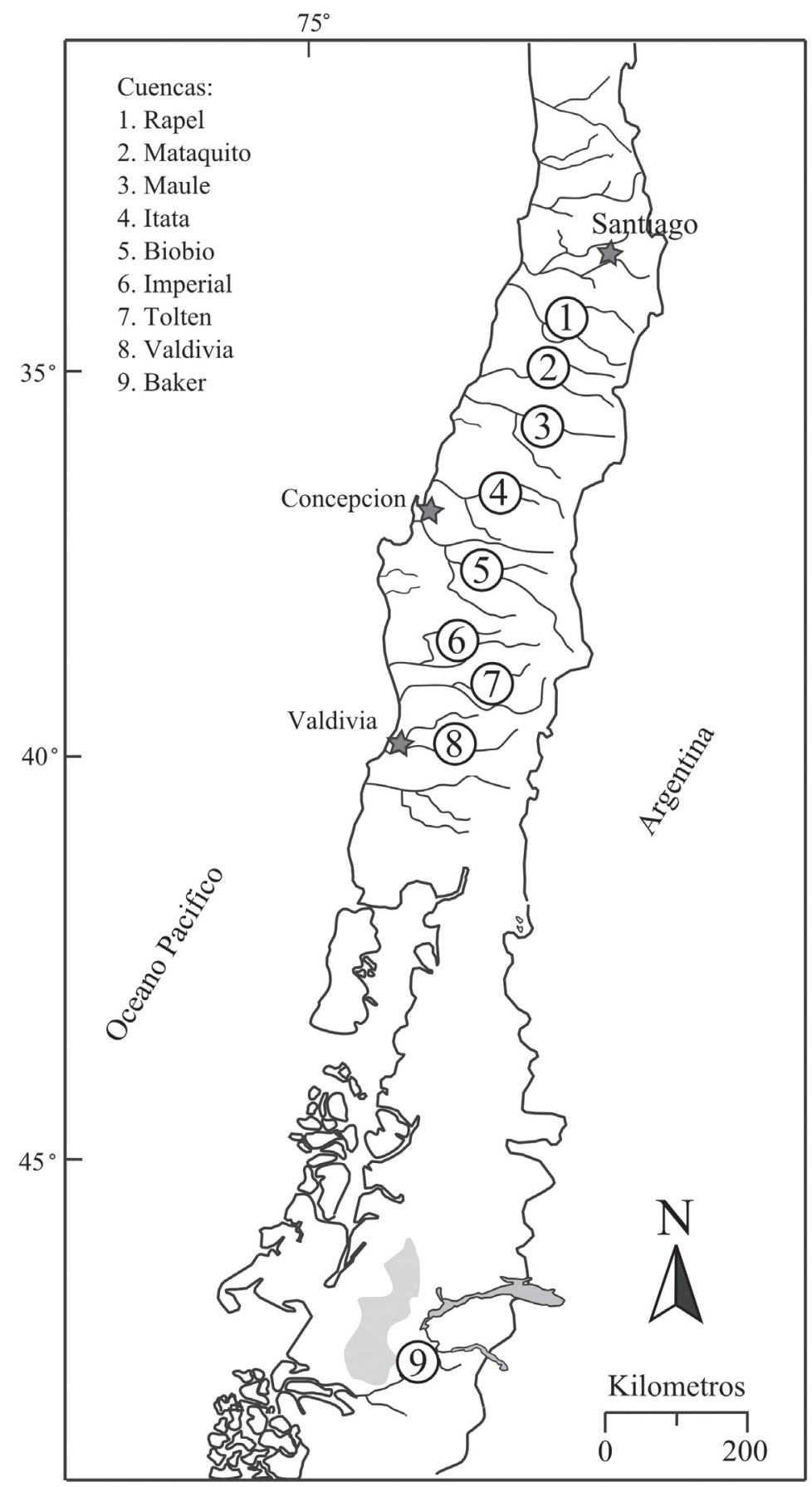

Figura 1. Cuencas con presencia de Diplomystes consideradas en este estudio.

FIgURE 1. Basins with presence of Diplomystes considered in this study. 
Actualmente, las tres especies de Diplomystes presentes en Chile se encuentran clasificadas En Peligro de Extinción (CONAMA 2008). Entre los factores responsables de la disminución de sus poblaciones destacan el deterioro del hábitat y la introducción de especies exóticas (Arratia 1983, 1987, Campos et al. 1998). Pese a que existen pocos estudios sobre aspectos básicos de la biología de los Diplomystes, se sabe que son carnívoros generalizados, consumidores de anélidos, moluscos, artrópodos y larvas de insectos acuáticos (Arratia 1987), pero se desconoce si la composición de la dieta cambia con los distintos estados de desarrollo o si existen diferencias a nivel poblacional.

La falta de estudios sobre la biología básica de Diplomystes, tales como su dieta, así como su relación con componentes ambientales, limita el conocimiento sobre la ecología de sus especies y poblaciones y consecuentemente dificulta la toma de decisiones para facilitar su conservación. Este aspecto es fundamental considerando que la gran mayoría de sus poblaciones están severamente amenazadas por el desarrollo hidroeléctrico de Chile, dado que son especies que utilizan casi exclusivamente hábitat ritrales (Habit et al. 2009). Por ello, en este trabajo analizamos la dieta de las distintas poblaciones de Diplomystes presentes en todo su rango distribucional en Chile con el fin de contribuir a la biología de un grupo de peces de gran importancia biológica $\mathrm{y}$ con un delicado estado de conservación.

\section{MATERIALES Y METODOS}

Se analizó el contenido estomacal de 175 individuos de Diplomystes spp provenientes de 9 cuencas hidrográficas en Chile desde el Río Rapel ( $\left.34^{\circ} \mathrm{S}\right)$ al Río Baker $\left(47^{\circ} \mathrm{S}\right)$ (Fig. 1). Estos individuos, colectados desde el año 1994 al 2009, fueron capturados mayoritariamente con pesca eléctrica (LR-24 Smith-Root equipment, USA), usando como valores máximos $350 \mathrm{~V} / 0.15 \mathrm{~A}$. Los ejemplares capturados fueron anestesiados, medidos, pesados, fijados en alcohol e individualizados con un número correlativo. Además se les inyectó alcohol en la zona ventral para reducir la degradación del contenido estomacal. Todo el material se encuentra depositado en la colección del Laboratorio de Ecología y Conservación de Peces del Centro EULA, Universidad de Concepción, Chile (códigos de los individuos en Tabla 1).

Para comparar la composición dietaria de los distintos estados ontogenéticos, cada individuo fue clasificado según su tamaño corporal en juvenil o adulto. Para ello, se emplearon los siguientes criterios. Para D. nahuelbutaensis la madurez sexual está descrita a los $11 \mathrm{~cm}$ de longitud total (Vila et al. 1996) y para D. camposensis a los $12 \mathrm{~cm}$ (Habit et al. 2009). Dado que solo se cuenta con tal información, se tomó como criterio utilizar como talla promedio de madurez sexual un largo total de $11,5 \mathrm{~cm}$ para todos los individuos en la muestra. Se establecieron así dos rangos de tallas o etarios: juveniles $<11,5 \mathrm{~cm}$ Longitud Total (LT) y adultos $\geq 11,5 \mathrm{~cm}$ LT (Tabla 1).

A cada individuo se le extrajo el estómago mediante disección abdominal, separando el tracto digestivo desde el esófago hasta los ciegos pilóricos. Se desechó el contenido intestinal por su avanzado estado de digestión. Cada estómago fue pesado e individualizado y su contenido fue vaciado y observado bajo estéreomicroscopio. Se analizó el contenido estomacal separando por ítems al menor nivel taxonómico posible. Se realizaron análisis numérico (conteo de ítems presa) y gravimétrico (peso de los ítems). Para el análisis gravimétrico se utilizó una balanza Shimadzu de $0,0001 \mathrm{~g}$ de precisión.

TABLA 1. Número de individuos analizados por cuencas hidrográficas, clasificados en juveniles $(<11.5 \mathrm{~cm}$ LT) y adultos $(\geq 11.5 \mathrm{~cm}$ LT).

TABLE 1. Number and code of individuals analyzed by hydrological basin and clasified as juveniles $(<11.5 \mathrm{~cm} \mathrm{LT})$ and adults $(\geq 11.5 \mathrm{~cm}$ LT).

\begin{tabular}{|c|c|c|c|c|}
\hline \multirow[b]{2}{*}{ Cuenca } & \multicolumn{3}{|c|}{$\mathrm{N}^{\circ}$ Individuos } & \multirow[b]{2}{*}{ Códigos individuos } \\
\hline & Juveniles & Adultos & Total & \\
\hline Rapel & 11 & 3 & 14 & DCa208, DTi86-92, DTi129-134 \\
\hline Mataquito & 28 & 1 & 29 & DLon166-176, DTe177-194 \\
\hline Maule & 14 & 7 & 21 & DMa144-151, DAch217-220, DAch230-238 \\
\hline Itata & 21 & 1 & 22 & $\begin{array}{l}\text { CCC01-09, DIt120-126, Ind.9, Ind.15 - Ind.18 } \\
\text { DBLL113-117, DDu110-112, DDu118, DDu119, }\end{array}$ \\
\hline Bíobío & 35 & 1 & 36 & $\begin{array}{l}\text { DBio127, DBio128, DLa198-207, Ind.1, Ind.2, } \\
\text { Ind.4, Ind.6-9, Ind.11-14, Ind.20-22 }\end{array}$ \\
\hline Imperial & 14 & - & 14 & DCau152-165 \\
\hline Toltén & 11 & 3 & 14 & DCol221-229, 6702B, RTdt, DTol07, DTo108 \\
\hline Valdivia & 16 & 1 & 17 & DEn209-213, Dc01-03, DCru240-248 \\
\hline Baker & 8 & - & 8 & DBk136-143 \\
\hline
\end{tabular}


Se construyeron dos matrices de ítems alimentarios. La primera fue expresada como número de presas por estómago de cada ítem, mientras que la segunda correspondió a una matriz gravimétrica expresada como el peso total de cada ítem presa. Cada uno de los peces analizados se consideró una réplica de un mismo sitio de muestreo, y los ítems se consideraron como las $\mathrm{n}$ variables en análisis.

Con el fin de conocer la estructura dietaria de los individuos juveniles y adultos de Diplomystes a lo largo de Chile, se utilizaron distintas aproximaciones. Primero, para analizar la importancia de los diferentes ítems presa en la dieta se estimaron los índices Q e IIN. El coeficiente alimentario numérico (Q) (Hureau 1970) representa el aporte relativo de cada ítem a la alimentación de los peces y se obtuvo del cálculo siguiente:

$\mathrm{Q}=$ Composición numérica $\mathrm{x}$ Frecuencia

A partir de lo cual se obtiene que:

$\mathrm{Q}<20=$ ítems terciarios

$\mathrm{Q}>21-200=$ secundarios

$\mathrm{Q}>201-10.000=$ primarios

Luego, el índice de importancia numérico (IIN) de cada ítem se calculó mediante la fórmula de Windell (1968)

$$
\mathrm{INN}=\sqrt{\% \text { composición numérica } x \text { frecuencia }}
$$

En segundo lugar, para determinar la amplitud del nicho trófico se estudiaron los índices: riqueza de especies S, índice de Diversidad de Shannon $H^{\prime}(\log 10)$ y equidad J, los que representan el grado de diversidad alimentaria.

Con el fin de determinar si existen patrones de similitud en la dieta de los ejemplares capturados entre las distintas cuencas hidrográficas, los datos de abundancia de ítem presas fueron transformados mediante raíz cuarta para reducir el peso de los ítems dominantes y luego se construyó una matriz de similitud basada en el índice de Bray-Curtis (Bray \& Curtis 1957), utilizando el programa PRIMER v.6 (Clarke \& Gorley 2006). Se efectuaron análisis exploratorios de los patrones de similitud dietaria entre cuencas mediante análisis de escalamiento multidimensional no métrico (nMDS). Con el propósito de evaluar la significancia de los factores ya mencionados se realizó un test de Análisis de Similitud (ANOSIM, Clarke 1993) de una vía, poniendo a prueba la significancia de las dietas de ambas tallas a través de las cuencas.

Para conocer cuáles ítems son los que más contribuyen a la disimilitud de la dietas, este análisis fue repetido utilizando solo los individuos juveniles, que fueron los de mayor representación en la muestra. Este análisis se realizó mediante un procedimiento de porcentajes de similitud
(SIMPER; v.6, Primer-E Ltd., Clarke \& Gorley 2005) tanto para la composición numérica como gravimétrica, pero sólo informamos los resultados de la primera, debido a que éstos fueron más resolutivos.

\section{RESULTADOS}

La mayoría de los individuos analizados correspondieron a juveniles (90,3 \% de la muestra), (Tabla 1). En las muestras analizadas, no se contó con adultos de las cuencas de los ríos Imperial y Baker. Del total de 175 estómagos analizados, el $29,4 \%$ se encontró vacío o con contenido no identificable. Todo el material estomacal observado fue de origen animal y no se encontraron evidencias de contenido vegetal en la dieta.

De acuerdo al nivel taxonómico al cual fueron identificadas las presas, la dieta estuvo constituida por un total de 34 ítems de origen animal, pertenecientes a 5 Clases (Tabla 2). La mayor parte de los ítems, tanto para juveniles como para adultos, correspondieron a insectos, principalmente en estados inmaduros. Los otros 4 grupos mayormente representados fueron el subphylum Crustacea, y los phyla Mollusca, Nematomorpha y Annelida. Los individuos juveniles destacaron por consumir preferentemente Chironomidae (Fig. 2). La dieta de algunos individuos adultos (mayor a 11,5 cm LT) se diferenció por consumir el ítem Aeglidae, tendencia más marcada en ejemplares de gran talla (Fig. 3).

La mayor diversidad en la dieta se encontró en individuos de las cuencas de los ríos Rapel y Baker, los que presentan una mayor equidad de los ítems presa (Fig. 4). En la cuenca del Rapel se encontró que los ítems Chironomidae y Sialidae corresponden a los ítems primarios, con un Q de 5434,78 y 1159,42 y con un IIN de $73,7 \%$ y $34 \%$ respectivamente. En tanto en la cuenca del Baker se determinó como ítem primario a Chironomidae $(\mathrm{Q}=1272,73)$, Tipulidae $(\mathrm{Q}=$ $242,42)$ y Diamphipnoidae $(\mathrm{Q}=242,42)$, y con un IIN de $35,7 \%, 15,6 \%$ y $15,6 \%$ respectivamente (Tabla 3 ). Solo en los individuos recolectados en la cuenca del rio Toltén no se registró el ítem Chironomidae como ítem primario. En esta cuenca el ítem primario correspondió exclusivamente a Simulidae, con un IIN de 62,7\%.

Al analizar la composición dietaria numérica, no se observaronn claros patrones intercuencas, aún cuando el análisis Anosim resulta significativo (ANOSIM, R Global = $0,102, p=0,001$ ), pero con un valor de $\mathrm{R}$ muy bajo, generado por el alto número de permutaciones posibles.

El análisis de escalamiento multi dimensional no métrico (nNMDS) de la composición numérica de la 
dieta de individuos juveniles (Fig. 5) distingue tres grupos significativos (ANOSIM, R Global $=0,26 ; \quad p=$ 0,001). El primero, denominado grupo I, está compuesto exclusivamente por individuos de la cuenca del Toltén, el grupo II, por ejemplares de la cuenca de Baker e Imperial, y el grupo III, por individuos de las cuencas del Rapel, Mataquito, Itata, Maule, Biobío y Valdivia. La dieta de los individuos del grupo I y II presentó un porcentaje de disimilitud de un 96,5\%, siendo Simulidae el ítem que mas aportó a esa disimilitud con un $34,5 \%$. La dieta de los peces de los grupos I y III presentó un porcentaje de disimilitud de un $94,28 \%$ y los ítems que mas aportaron a esta disimilitud fueron Simulidae con un 29,18\% y Chironomidae con un $27,11 \%$. Entre los grupos II y III se observó un porcentaje de disimilitud de un $81,59 \%$ y el ítem que mas aportó a esto fue Chironomidae con un 31,86\% (Tabla 4).

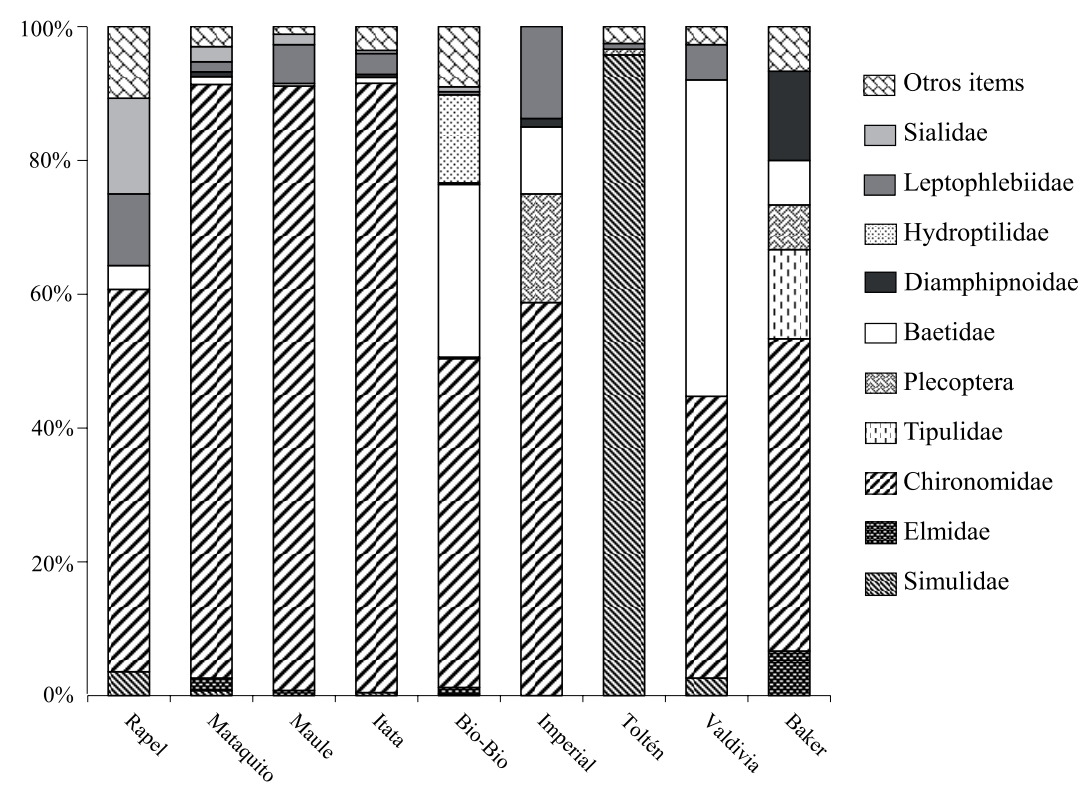

FIgURA 2. Composición numérica de la dieta de juveniles de Diplomystes por cuenca hidrográfica.

FIGURE 2. Composition of diet of juvenile Diplomystes by hydrographic basin.

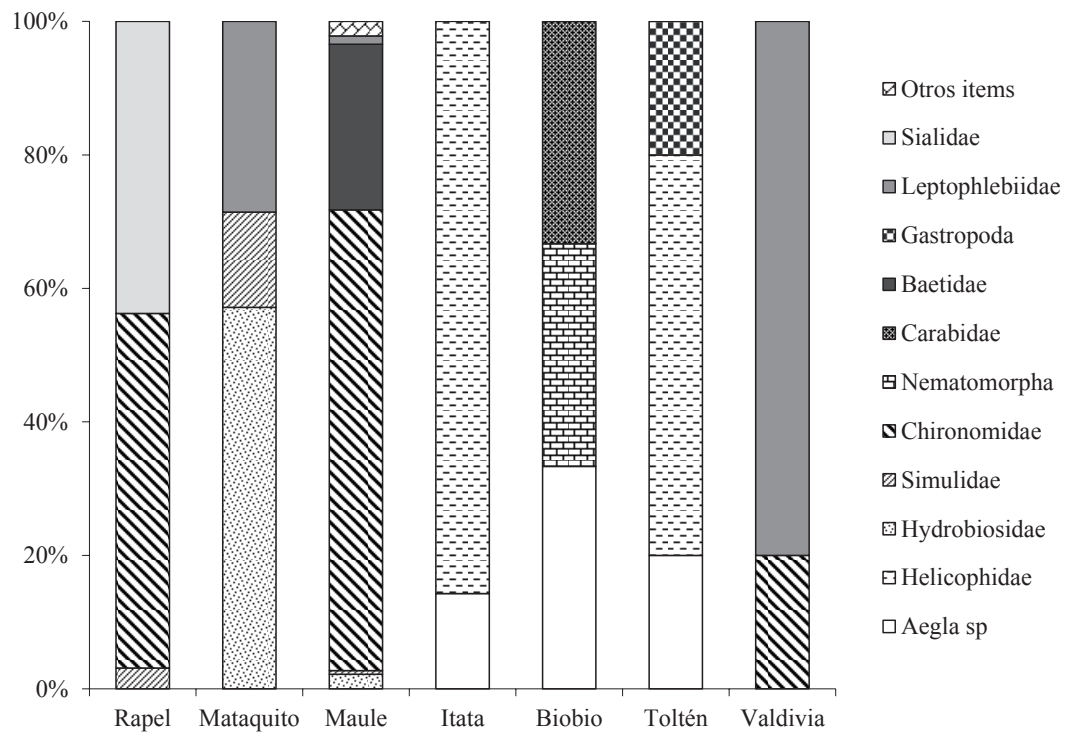

FiguRa 3. Composición numérica de la dieta de adultos de Diplomystes por cuenca hidrográfica.

FIGURE 3. Numeric composition of adult diet Diplomystes by hydrographic basin. 
Dieta de Diplomystes de Chile: Beltrán-Concha et AL.

TABla 2. Items consumidos por juveniles (J) y adultos (A) de Diplomystes en las nueve cuencas estudiadas.

TABLE 2. Items consumed by juveniles (J) and adults (A) of Diplomystes in the nine basins studied.

\begin{tabular}{|c|c|c|c|c|c|c|c|c|c|c|c|c|c|c|c|c|c|c|c|}
\hline & \multirow[b]{2}{*}{ Item presa } & \multicolumn{2}{|c|}{ Rapel } & \multicolumn{2}{|c|}{ Mataquito } & \multicolumn{2}{|c|}{ Maule } & \multicolumn{2}{|c|}{ Itata } & \multicolumn{2}{|c|}{ Biobío } & \multicolumn{2}{|c|}{ Imperial } & \multicolumn{2}{|c|}{ Toltén } & \multicolumn{2}{|c|}{ Valdivia } & \multicolumn{2}{|c|}{ Baker } \\
\hline & & $\mathrm{J}$ & $\mathrm{A}$ & $\mathrm{J}$ & A & $\mathrm{J}$ & A & $\mathrm{J}$ & A & $\mathrm{J}$ & $\mathrm{A}$ & $\mathrm{J}$ & $\mathrm{A}$ & $\mathrm{J}$ & A & $\mathrm{J}$ & A & $\mathrm{J}$ & A \\
\hline Crustácea & 1. Aeglidae & & & & & & & & $\mathrm{X}$ & & $\mathrm{X}$ & & & & $\mathrm{X}$ & & $\mathrm{X}$ & & \\
\hline Unirramia & 2. Huevos de insecto & & & & & & $\mathrm{X}$ & & & $\mathrm{X}$ & & & & & & & & & \\
\hline (Insecta) & 3. Helicophidae & & & & & & & & $\mathrm{X}$ & & & & & $\mathrm{X}$ & $\mathrm{X}$ & & & & \\
\hline & 4. Hydrobiosidae & $\mathrm{X}$ & & $\mathrm{X}$ & $\mathrm{X}$ & $\mathrm{X}$ & $\mathrm{X}$ & & & $\mathrm{X}$ & & & & & & & & & \\
\hline & 5. Hydropsychidae & $\mathrm{X}$ & & & & & & $\mathrm{X}$ & & $\mathrm{X}$ & & & & $\mathrm{X}$ & & & & $\mathrm{X}$ & \\
\hline & 6. Pupa trichoptera & & & & & $X$ & & & & & & & & & & & & & \\
\hline & 7. Anamalopsychidae & & & & & & $\mathrm{X}$ & & & $\mathrm{X}$ & & & & & & & & & \\
\hline & 8. Pupa Trichoptera & & & & & $\mathrm{X}$ & & & & & & & & & & & & & \\
\hline & 9. Polycentropodidae & & & & & $X$ & $X$ & & & & & & & & & & & & \\
\hline & 10. Hydroptilidae & & & & & & & & & $\mathrm{X}$ & & & & & & & & & \\
\hline & 11. Simulidae & $\mathrm{X}$ & $\mathrm{X}$ & $\mathrm{X}$ & $X$ & & $X$ & $\mathrm{X}$ & & $\mathrm{X}$ & & & & $\mathrm{X}$ & & $\mathrm{X}$ & & & \\
\hline & 12. Chironomidae & $\mathrm{X}$ & $\mathrm{X}$ & $\mathrm{X}$ & & $\mathrm{X}$ & $\mathrm{X}$ & $\mathrm{X}$ & & $\mathrm{X}$ & & $\mathrm{X}$ & & & & $\mathrm{X}$ & & $\mathrm{X}$ & \\
\hline & 13. Tipulidae & & & & & & & & & $\mathrm{X}$ & & & & & & & & $\mathrm{X}$ & \\
\hline & 14. Pupa diptero & & & & & & $\mathrm{X}$ & $\mathrm{X}$ & & & & & & & & & & & \\
\hline & 15. Adulto Diptero & $\mathrm{X}$ & & $\mathrm{X}$ & & & & & & & & & & & & & & & \\
\hline & 16. Empididae & & & & & & $\mathrm{X}$ & & & & & & & & & & & & \\
\hline & 17. Elmidae & & & $\mathrm{X}$ & & $\mathrm{X}$ & $\mathrm{X}$ & & & $\mathrm{X}$ & & & & & & & & $\mathrm{X}$ & \\
\hline & 18. Curculionidae & & & & & & $X$ & & & & & & & & & & & & \\
\hline & 19. Carabidae & & & & & & & & & & $\mathrm{X}$ & & & & & & & & \\
\hline & 20. Coleoptero indet. & & & & & $\mathrm{X}$ & & & & & & & & & & & & & \\
\hline & 21. Ephydridae & & & & & & & & & & & & & & & $\mathrm{X}$ & & & \\
\hline & 22. Diamphipnoidae & & & $\mathrm{X}$ & & & & $\mathrm{X}$ & & $\mathrm{X}$ & & $X$ & & & & & & $\mathrm{X}$ & \\
\hline & 23. Gripopterygidae & & & $\mathrm{X}$ & & & & $\mathrm{X}$ & & $\mathrm{X}$ & & & & $\mathrm{X}$ & & & & & \\
\hline & 24. Notonemouridae & & & & & & & & & $\mathrm{X}$ & & & & & & & & & \\
\hline & 25. Plecoptera n.i & & & & & & & & & & & $\mathrm{X}$ & & $\mathrm{X}$ & & & & $\mathrm{X}$ & \\
\hline & 26. Nesameletidae & & & & & & & $\mathrm{X}$ & & & & & & & & & & & \\
\hline & 27. Baetidae & $\mathrm{X}$ & & $\mathrm{X}$ & & $\mathrm{X}$ & $X$ & $\mathrm{X}$ & & $\mathrm{X}$ & & $\mathrm{X}$ & & & & $\mathrm{X}$ & & $\mathrm{X}$ & \\
\hline & 28. Leptophlebiidae & $\mathrm{X}$ & & $\mathrm{X}$ & $\mathrm{X}$ & $\mathrm{X}$ & $X$ & $\mathrm{X}$ & & $\mathrm{X}$ & & $\mathrm{X}$ & & $\mathrm{X}$ & & $\mathrm{X}$ & & & \\
\hline & 29. Amelotopsidae & & & & & & & & & $\mathrm{X}$ & & & & & & & & & \\
\hline & 30. Caenidae & & & & & & & & & $\mathrm{X}$ & & & & & & & & & \\
\hline & 31. Sialidae & $\mathrm{X}$ & $\mathrm{X}$ & $\mathrm{X}$ & & $\mathrm{X}$ & & $\mathrm{X}$ & & $\mathrm{X}$ & & & & & & & & & \\
\hline Mollusca & 32. Adulto Gastropoda & & & & & & & & & & & & & & $\mathrm{X}$ & & & & \\
\hline Nematomorpha & 33. Gordidae & & & & & & & & & & $\mathrm{X}$ & & & & & & & & \\
\hline Annelida & 34. Oligochaeta & & & & & & & $X$ & & & & & & $\mathrm{X}$ & & & & & \\
\hline
\end{tabular}




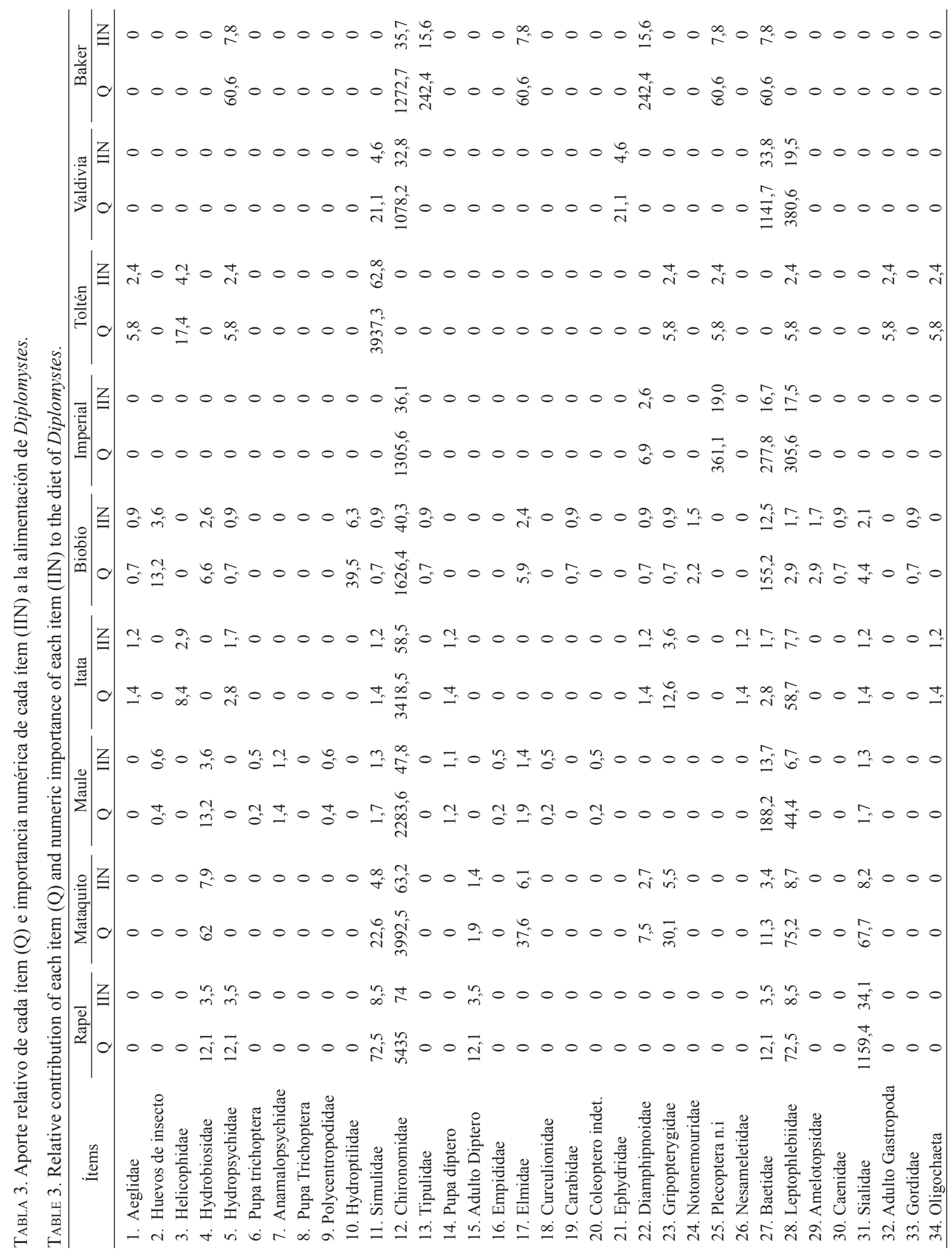


Dieta de Diplomystes de Chile: Beltrán-Concha et AL.

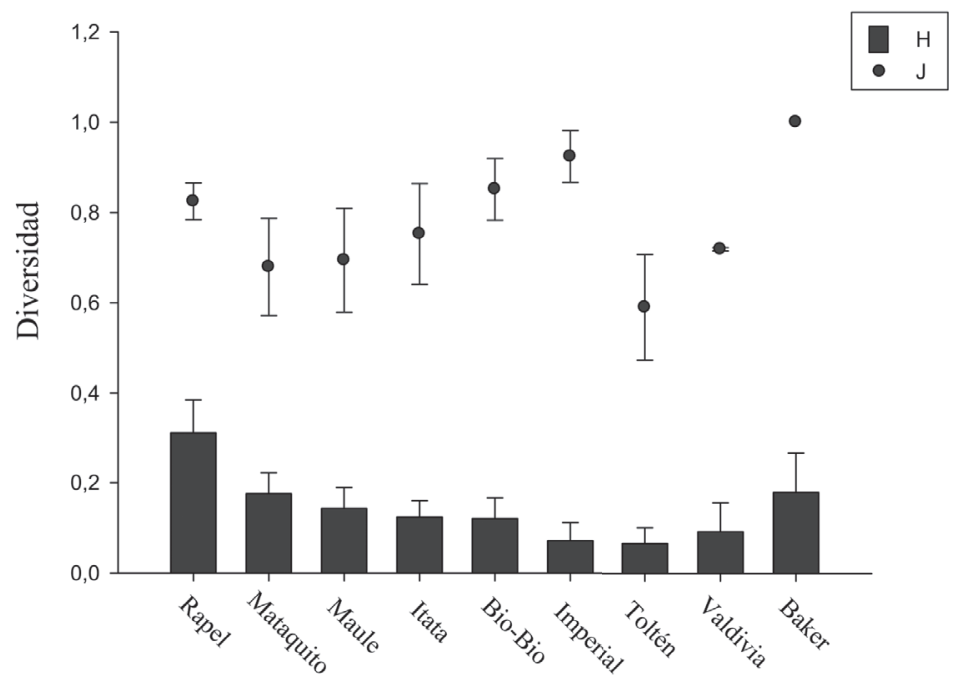

Figura 4. Diversidad dietaria de Diplomystes juveniles a lo largo de Chile. H, índice de diversidad de Shannon, J, equidad. Las barras indican error estándar.

FIGURE 4. Dietary diversity of juveniles of Diplomystes in Chile. H, Shannon diversity, J, equity. Bar lines show standard deviation.

TABla 4. Porcentajes de disimilitud entre pares de grupos (en negrita), abundancia media de los ítems presa por grupo y disimilitud promedio entre pares de grupos. En paréntesis se indica el porcentaje de contribución en que cada ítem presa explica las diferencias dietarias del total de peces por grupo (basado en el análisis SIMPER de una vía para composición numérica de la dieta). Notar que todos los pares de grupos presentan diferencias significativas, según los análisis pareados de ANOSIM.

TABLE 4. Pairwise dissimilitude percentage among groups (in bold), mean prey items abundance per group, and average dissimilitude among pair of groups. Contribution in which each prey item explains dietary differences of the total number of fishes per group is shown in parenthesis (based on the one-way SIMPER analysis for numeric composition of diet).

\begin{tabular}{|c|c|c|c|c|c|c|}
\hline \multirow[b]{2}{*}{ Item } & \multicolumn{3}{|c|}{ Abundancia media } & \multicolumn{3}{|c|}{ Disimilitud media y contribución (\%) } \\
\hline & I & II & III & $\begin{array}{l}\text { I v/s II } \\
\mathbf{9 6 , 4 7}\end{array}$ & $\begin{array}{c}\text { I v/s III } \\
\mathbf{9 4 , 2 8}\end{array}$ & $\begin{array}{c}\text { II v/s III } \\
\mathbf{8 1 , 5 9}\end{array}$ \\
\hline Larva Chironomidae & 0 & 0,5 & 1,16 & $14,87(15,41)$ & $25,56(27,11)$ & $25,99(31,86)$ \\
\hline Larva Leptophlebiidae & 0,13 & 0,23 & 0,34 & $7,95(8,25)$ & $9,06(9,61)$ & $11,17(13,69)$ \\
\hline Larva Baetidae & 0,00 & 0,31 & 0,19 & $9,66(10,02)$ & $3,74(3,97)$ & $10,79(13,23)$ \\
\hline Plecoptera & 0,13 & 0,30 & 0,00 & $11,91(12,35)$ & $4,06(4,31)$ & $8,15(9,98)$ \\
\hline Diamphipniodae & 0 & 0,14 & 0,05 & $3,46(3,59)$ & - & $4,26(5,22)$ \\
\hline Sialidae & 0 & 0 & 0,19 & - & $3,37(3,57)$ & $3,49(4,28)$ \\
\hline Gripopterygidae & 0,13 & 0 & 0,10 & $4,98(5,17)$ & $5,82(6,18)$ & $2,69(3,29)$ \\
\hline Simulidae & 1,27 & 0 & 0,09 & $33,33(34,55)$ & 27,51 (29.18) & $2,63(3,22)$ \\
\hline Hydropsychidae & 0,13 & 0,05 & 0,00 & $3,77(3,91)$ & $2,58(2,74)$ & - \\
\hline Elmidae & 0 & 0,05 & 0,12 & - & $2,54(2,70)$ & $3,39(4,14)$ \\
\hline
\end{tabular}




\section{DISCUSIÓN}

Este es uno de los primeros trabajos que analiza un aspecto biológico de Diplomystes a través de su amplia distribución latitudinal en Chile. La mayoría de los trabajos se han realizado en áreas acotadas a cuencas o zonas determinadas (e.g. Arratia 1983; Habit 2005). Dado el amplio rango geográfico que abarca este estudio (entre los $34^{\circ}-47^{\circ}$ Lat. Sur), la muestra de individuos analizados es latitudinalmente significativa. Sin embargo, debido al mayor número de ejemplares juveniles analizados, este estudio es más representativo de la dieta de juveniles de las poblaciones de Diplomystes presentes en Chile.

Un resultado interesante es que Diplomystes, cuando alcanza talla de adulto, presenta la capacidad de comer Aeglidos, ítem que es importante por biomasa y que diferencia a adultos de juveniles. No todos los adultos consumieron Aegla (e.g. aquellos provenientes de las cuencas Rapel y Maule), pero la tendencia observada fue que los de mayor talla tuvieron este ítem alimentario en sus estómagos. En tanto, otros adultos que no contenían Aegla en sus estómagos presentaron idéntica dieta con los individuos juveniles. Desafortunadamente, en la muestra de individuos del río Baker no se contaba con ejemplares adultos, un aspecto interesante de haber sido analizado dada la aparente ausencia de Aegla en esta cuenca (Perez-Lozada et al. 2008; Retamal \& Moyano 2010). Resultaría de gran interés investigar a futuro en que basan su dieta los adultos de Diplomystes de la cuenca del Baker.

A pesar del amplio rango latitudinal y alto número de individuos analizados entre cuencas, no se observaron diferencias significativas entre las distintas dietas, lo que podría atribuirse a dos aspectos diferentes, no necesariamente excluyentes entre sí. En primer lugar, que la identificación taxonómica de los ítems presa a altos niveles de jerarquía taxonómica (e.g. Familia) no esté dando cuenta de la diversidad dietaria de Diplomystes. Así por ejemplo, es posible que los quironómidos consumidos en la cuenca del Rapel no sean las mismas especies o géneros consumidos en la cuenca del Baker y que sin embargo, en este estudio son considerados de valor idéntico en el análisis. A pesar de su gran diversidad, enorme abundancia y gran relevancia ecológica, estas pequeñas larvas acuáticas son uno de los grupos menos estudiados en Chile por lo que se hace difícil una identificación a nivel de especie (Valdovinos 2006). En segundo lugar, que los Diplomystes tengan una dieta bentófaga generalista, tal como lo señaló Arratia (1987), por lo cual están consumiendo grupos funcionales de macroinvertebrados bentónicos que tienden a ser los mismos a nivel taxonómico alto (e.g. Familia). Independientemente del nivel taxonómico de los ítems presa, los resultados indican que los peces del género Diplomystes consumen los mismos grupos funcionales de invertebrados acuáticos a lo largo de su distribución latitudinal en Chile, y sugieren que la diferencia a nivel específico de macroinvertebrados bentónicos como quironómidos realmente no reflejaría una conducta alimentaria diferente por parte de los peces.

Dadoqueel género Diplomystes es un grupo depeces primitivo y con escasa diferenciación morfológica interespecífica, es esperable que no muestren especializaciones alimentarias. En este contexto, lo esperable es que las potenciales diferencias en la dieta estuvieran dadas por la oferta de alimento más que por la selección que hicieran los individuos de sus presas. En este estudio no fue considerado el análisis de la oferta dietaria, pero resultaría necesario incluirlo en estudios posteriores, para definir si son peces realmente generalistas o tienen una especialización por determinados grupos funcionales.

El hecho de que los individuos juveniles estén consumiendo preferentemente quironómidos (excepto en el Toltén cuyo principal ítem fue Simulidae), sugiere fuertemente un uso de hábitat similar para todas las poblaciones estudiadas a lo largo del gradiente latitudinal. Tanto Chironomidae como Simulidae, que habitan en la zonas ritrales, son preferentemente detritívoros y se encuentran asociados a sustratos con abundante perifiton (R. Figueroa com. pers). Este es el tipo de hábitat que tanto Arratia (1983) como Habit et al. (2009) describieron como hábitat preferente de los juveniles de menor talla de D. chilensis y $D$. camposensis, respectivamente. Este aspecto resulta en que la diferenciación dietaria de las poblaciones de Diplomystes se manifieste, al menos cualitativamente, sólo en su estado adulto.

\section{AGRADECIMIENTOS}

Agradecemos a Alfonso Jara y Alvaro Zuñiga por su ayuda en terreno y a Jorge González por su apoyo en la identificación de las muestras en etapas tempranas de este trabajo. Este estudio fue financiado por el proyecto Fondecyt 1080082 y Colbun S.A.

\section{BIBLIOGRAFÍA}

Arratia, G. 1983. Preferencias de Hábitat de peces siluriformes de aguas continentales de Chile (Fam. Diplomystidae y Trichomycteridae). Studies on Neotropical Fauna and Environment, 18(4): 217-237.

Arratia, G. 1987. Description of the primitive family Diplomystidae (Siluriformes, Teleostei, Pisces): morphology, taxonomy and plylogenetic implications. Bonner Zoologische Monographien, 24: 1-120.

Arratia, G. 1992. Development and variation of the suspensorium 
of primitive catfishes (Teleostei: Ostariophysi) and their phylogenetic relationshisps. Bonner Zoologische Monographien, 32: 1-149.

BRAY, J.R. \& CURTIS, J.T. 1957. An ordination of the upland forest communities of southern Wisconsin. Ecological Monographs, 27:325-349.

Campos, H. 1985. Distribution of fishes in the Andean Rivers in the South of Chile. Archives Hydrobiology, 104(2): 169-191.

Campos, H., Dazarola, G., Dyer, B., Fuentes, L., Gavilán, J.F., Huaquin, L., Martinez, G., Meléndez, R., Pequeño, G., Ponce, F., Ruiz, V.H., Sielfeld, W., Soto, D., Vera, R. \& VILA, I. 1998. Categorías de Conservación de peces nativos de aguas continentales de Chile. Boletin del Museo Nacional de Historia Natural, Santiago de Chile, 47: 101122.

Clarke, K.R. 1993. Non-parametric multivariate analyses of changes in community structure. Australian Journal of Ecology, 18: 117-143.

Clarke, K.R. \& Gorley, R.N. 2006. PRIMER v.6: User Manual/ Tutorial. PRIMER-E Ltda., Playmouth, UK. 192 PP.

Conama. 2008. URL: http://www.sernapesca.cl/index. php?option $=$ com_remository\&Itemid $=246 \&$ func $=$ select $\&$ $\mathrm{id}=294$ \&orderby $=2$ \&page $=3$.

Dyer, B. 2000. Systematic review and biogeography of the freshwater fishes of Chile. Estudios Oceanologicos, Chile 19: 77-98.

GRANDE, L. 1987. Redescription of †Hypsidoris farsonensis (Teleostei: Siluriformes), with a reassessment of its phylogenetic relationships. Journal of Vertebrate Paleontology, 7: 24-54.

Habit, E. 1994. Contribución al conocimiento de la fauna íctica del Río Itata. Boletín de la Sociedad de Biología de Concepción, Chile, 65: 143-147.

Habit, E. 2005. Aspectos de la biología y hábitat de un pez endémico de Chile en peligro de extinción. Interciencia, 30: 8-11.

Habit, E., Jara, A., Colin, N., Oyanedel, A., Victoriano, P., Gonzalez, J. \& Solis-Lufí, K. 2009. Threatened fishes of the world: Diplomystes camposensis Arratia, 1987 (Diplomystidae). Environmental Biology of Fishes, 84: 393-394.

Hureau, J.C. 1970. Biologie compare de quelques poisons antarctiques (Nototheniidae). Bulletin de l'Institute Ochnographique, Monaco, 68: 1-244.
Jara, C., Rudolph, E. \& GonZalez, E. 2006. Estado de conocimiento de los crustáceos malacostracos dulceacuícolas de Chile. Gayana 70: 72-81.

Moya, C., Valdovinos, C., Moraga, A., Romero, F., Debels, P. \& Oyanedel, A. 2009. Patrones de distribución espacial de ensambles de macroinvertebrados bentónicos de un sistema fluvial Andino Patagónico. Boletín del Museo Nacional de Historia Natural, Santiago de Chile, 82: 425-442.

Muñoz Ramírez, C., Jara, A., Beltrán-Concha, M., ZúNigaReinoso, A., Victoriano, P. \& Habit, E. 2010. Distribución de la familia Diplomystidae (Pisces: Siluriformes) en Chile: Nuevos Registros. Boletín de Biodiversidad de Chile, 4: 6-17.

Pérez-Lozada, M., Bond-Buckup, G., Jara, C.G. \& Crandall, K.A. 2008. Conservation Assessment of Southern South American Freshwater Ecoregions on the Basis of the Distribution and Genetic Diversity of Crabs from the Genus Aegla. Conservation Biology, Volume 23, No. 3, 692-702.

De PinNa, M.C.C. 1998. Phylogenetic relationships of Neotropical Siluriformes (Teleostei: Ostariophysi): historical overview and synthesis of hypotheses. En: Phylogeny and classification of Neotropical fishes (Eds. Malabarba, L. R., R. E. Reis, R. P.Vari, Z. M. S. Lucena \& C. A. S. Lucena, pp. 279-330. EDIPUCRS.

Retamal, M. A. \& Moyano H.I. 2010. Zoogeografía de los crustáceos decápodos chilenos marinos y dulceacuícolas. Latin American Journal of Aquatic Research, 38(3): 302328.

Sullivan, J.P., Lundberg, J.G. \& Hardman, M. 2006. A phylogenetic analysis of the major groups of catfishes (Teleostei: Siluriformes) using rag1 and rag2 nuclear gene sequences. Molecular Phylogenetics and Evolution, 41: 636-662.

VALDOVINOS, C. 2006. Invertebrados dulceacuícolas. Biodiversidad de Chile patrimonio y desafíos. CONAMA: 204-225.

Vila, I., Contreras, M., Fuentes, L. 1996. Reproducción de Diplomystes nahuelbutaensis Arratia 1987 (Pisces: Diplomystidae). Gayana Oceanol. 4(2): 129-137.

Windell, J. T. 1968. Food analysis and rate of digestión. En: Methods for assessment of fish production in freshwaters (Ed. Ricker W.E.), 3: 197-203. Editorial IBP Handbook.

Recibido: 19.01.12

Aceptado: 08.08.12 